\title{
Aplicación de una metodología mixta para la selección de materiales resistentes a la corrosión en medios ácidos y salinos
}

\author{
Application of mixed method for selection of corrosion resistant materials in acidic and \\ saline environments.
}

\section{Aplicação de uma metodologia mista para a seleção de materiais resistentes à corrosão em médios ácidos e salinos}

\author{
Andrey Felipe Mahecha-Gómez', Claudia Patricia Mejía-Villagrán², Jhon Jairo Olaya-Flórez ${ }^{3}$
}

Forma de citar: A. F. Mahecha, C. P. Mejía, J. J. Olaya, “Aplicación de una metodología mixta para la selección de materiales resistentes a la corrosión en medios ácidos y salinos", Respuestas, vol. 20, no. 1, pp. 112 - 124, 2015.

Recibido:

20 de Agosto 2014

Aceptado:

10 de Noviembre 2014

\section{Resumen}

Objetivo: En este artículo se presenta una metodología mixta de selección de materiales para determinar qué material presenta la mejor relación entre resistencia a la corrosión, propiedades mecánicas y costos. Metodología: El trabajo se llevó a cabo utilizando pruebas potenciodinámicas de extrapolación (Tafel) e impedancia electroquímica (EIS), sometiendo las muestras a medios corrosivos de $\mathrm{NaCl}(3 \%)$ y $\mathrm{H}_{2} \mathrm{SO}_{4}(5 \%)$. Las muestras utilizadas fueron el cerámico Zirconia no sinterizada y los aceros AISI 304L, AISI 316 y AISI 316L. Finalmente se aplicó una metodología de selección de materiales para escoger el material que presenta mejor comportamiento en este tipo de medios. Resultados: Como resultado, para las pruebas de extrapolación Tafel con 5\% de ácido sulfúrico (H2SO4), se obtuvo que el acero AISI 316 presenta la corriente de corrosión más baja y una pasivación a mayor potencia. En el medio electrolítico con $3 \%$ de $\mathrm{NaCl}$, se observa que los valores de potencial de corrosión más altos se presentan en los aceros inoxidables, evidenciándose una menor velocidad de corrosión en los aceros de la serie 316. Conclusiones: Como resultado se obtuvo que el acero AISI 316 utilizado presenta el mejor comportamiento de resistencia a la corrosión en los medios Medios ácidos y salinos.

Palabras claves: Corrosión, Medios ácidos y salinos, Metodología mixta, Selección de materiales.
Abstract
Objective: This article presents a mixed materials selection methodology is presented to determine which material has the best balance between corrosion resistance, mechanical properties and costs. Methods: The work was realized using potentiodynamic tests of extrapolation (Tafel) and electrochemical impedance (EIS), subjecting the samples to corrosive media of $\mathrm{NaCl}(3 \%)$ and $\mathrm{H} 2 \mathrm{SO} 4(5 \%)$. The samples used were zirconia, steel AISI 304L, AISI 316 and AISI 316L. Finally a mixed methodology was applied in the materials selection to choose the material that has better comportment in this type of electrolyte. Results: For Tafel extrapolation test with 5\% sulfuric acid (H2SO4) was obtained that AISI 316 steel which has the lowest current corrosion and passivation at higher power. In the electrolytic medium with $3 \% \mathrm{NaCl}$, shows that higher values for corrosion potential present in stainless steels, showing a lower corrosion rate in the steels of the 316 series. Conclusions: The results showed that steel AISI 316 used 
presents the best performance to corrosion resistance in saline and acid.

Keywords: Corrosión, Acidic and saline environments, mixed methodology, Selection of materials.

\section{Resumo}

Objetivo: Neste artigo se expõe uma metodologia mista de seleção de materiais para determinar que material presenta a melhor relação entre resistência à corrosão, propriedades mecânicas e custos. Metodologia: $\mathrm{O}$ trabalho se realizou utilizando provas potencio-dinâmicas de extrapolação (Tafel) e impedância eletroquímica (EIS), sometendo as amostras a médios corrosivos de $\mathrm{NaCl}(3 \%)$ e $\mathrm{H}_{2} \mathrm{SO}_{4}(5 \%)$. As amostras utilizadas foram a cerâmica Zircônia não sinterizada e os aços AISI 304L, AISI 316 e AISI 316L. Finalmente, se aplicou uma metodologia de seleção de materiais para escolher o material que apresenta melhor comportamento neste tipo de médios. Results: For Tafel extrapolation test with $5 \%$ sulfuric acid (H2SO4) was obtained that AISI 316 steel which has the lowest current corrosion and passivation at higher power. In the electrolytic medium with $3 \%$ $\mathrm{NaCl}$, shows that higher values for corrosion potential present in stainless steels, showing a lower corrosion rate in the steels of the 316 series. Conclusões: Como resultado se obteve que o aço AISI 316 utilizado apresenta o melhor comportamento de resistência à corrosão nos médios salino e ácido.

Palavras-chave: Corrosão, Médios ácidos e salinos, metodologia mista, Seleção de materiais.

\section{Introducción}

Debido a los avances tecnológicos, en la actualidad se cuenta con gran número de materiales para aplicaciones específicas, por lo cual se hace necesario, el establecimiento de estrategias y/o métodos que permitan la selección de un material apropiado frente a cierta aplicación, teniendo en cuenta aspectos tales como composición del material, procesos de conformado, condiciones de trabajo y relación entre costo y desempeño.

Junto al desgaste y los cambios de temperatura, la corrosión constituye un problema de enormes proporciones que necesita ser controlado, particularmente en el sector industrial metalmecánico, energético, naviero y aeronáutico. La corrosión es la fuente principal de la degradación y posterior pérdida de la mayoría de los materiales. Debido al desarrollo industrial y al empleo de combustibles con diferentes tipos de aditivos; se ha generado una nueva dinámica atmosférica, causando que los aceros y otros metales expuestos a ella, se degraden más rápidamente [1], [2]. Se estima que las pérdidas económicas generadas por la corrosión, en el caso de Colombia; ascienden a más de 26 mil millones de pesos (4\% del PIB) [3]. Para contrarrestar ese efecto, recientemente se tiene un buen número de materiales tipo recubrimientos relativamente inertes, con buenas propiedades anticorrosivas que protegen la superficie del material (generalmente metálico).

En el caso de los aceros inoxidables se observa el fenómeno de auto pasivación espontánea que se presenta cuando una superficie limpia se expone a un entorno que pueda proveer de suficiente oxígeno para formar la capa superficial de óxido rico en cromo. Esto ocurre automática e instantáneamente, siempre que haya suficiente oxígeno disponible en la superficie del acero. No obstante la capa pasiva, aumenta de grosor durante algún tiempo después de su formación inicial [4]. Ciertas condiciones naturales, como el contacto con el aire o con agua aireada, crearán y mantendrán 
No. 1

Enero - Junio 2015 ISSN 0122-820X

PP: $112-124$

114 la condición pasiva de la superficie frente a la corrosión. En el caso específico de los aceros inoxidables austeníticos como los que se trabajarán en este artículo, se han encontrado mejores propiedades en comparación con otras familias de este tipo material con una elevada resistencia a los diferentes mecanismos de corrosión, una relativa facilidad de trasformación, así como un buen comportamiento a bajas y altas temperaturas [5].

El grupo de materiales de estudio son los aceros inoxidables austeníticos de la serie AISI 316L, 316,304L, además del cerámico zirconia. La zirconia es un material polimórfico que se puede encontrar en tres estructuras cristalinas dependiendo de la temperatura: monoclínica (m) a temperatura ambiente, tetragonal $(\mathrm{t})$ por encima de $1170^{\circ}$ $\mathrm{C}$, y cúbica (c) más allá de $2370^{\circ} \mathrm{C}$. El uso de esta cerámica como material de carga, se basa en sus propiedades mecánicas útiles combinadas con una excelente estabilidad química [4].

El acero AISI316L es un acero inoxidable de tipo austenítico, con importantes aplicaciones biomédicas. Este acero es una variante del AISI3 16 con un contenido de carbono inferior, menor límite elástico y también menor resistencia a la tracción. Ofrece en cambio mejor soldabilidad y menor probabilidad de aparición de corrosión entre las uniones o corrosión intergranular [6]. Se utiliza ampliamente como material de implante para fabricar dispositivos como articulaciones artificiales, placas óseas, soportes y prótesis de cadera. Tiene buenas propiedades mecánicas, alta resistencia a la corrosión y un coste relativamente bajo en comparación con otros biomateriales metálicos [7].

El acero AISI 304L es un acero inoxidable austenítico de uso general con una estructura FCC. Es esencialmente no magnético en estado recocido y sólo puede endurecerse en frío. Adiciones suplementarias de cromo $\mathrm{y}$ níquel otorgan una mejor resistencia al calor [8]. Este contiene $18.5 \% \mathrm{Cr}, 9.5 \% \mathrm{Ni}$, $2.0 \% \mathrm{Mn}, 1.0 \% \mathrm{Si}, 0.03 \% \mathrm{C}$. Al ser expuesto a atmósferas simuladas de gasificación de carbón en un autoclave de multi-muestra para un máximo de 1.000 horas a $600^{\circ} \mathrm{C}$, en atmósferas de oxígeno y sulfuro de carbono, tiene crecimiento las escalas de superficie y aumenta la corrosión interna, así como también presenta cambios en la composición y morfología de los productos de corrosión [9]

La metodología de Michael Ashby [10], [11] es una guía en el proceso de selección de un material. En las diversas fases de diseño, contempla los atributos que se requieren por medio de cartas de selección. Esta metodología contempla la maximización o minimización de un valor de índice de desempeño que hace parte de una función objetivo, la cual está directamente relacionada con las propiedades físico-químicas de estudio.

Esta metodología contempla cuatro pasos: Traducción, Filtrado, Clasificación y Documentación. La metodología de selección con ayuda de bases de datos consiste en la escogencia del material mediante dichas bases que se dividen básicamente en dos categorías, numéricas y literarias o de referencias bibliográficas. Dentro de las más importantes bases de datos están el banco de datos de la ASTM, la SAE, la ASM, la AISI, la NASA, etc. [11]. Estas bases de datos son el resultado de investigaciones en ensayos de materiales.

En el presente trabajo se evaluó la resistencia a la corrosión de 3 aceros inoxidables y uno cerámico. Se les realizaron a las probetas pruebas de dureza, ensayos de corrosión potenciodinámica o extrapolación Tafel e impedancia electroquímica EIS. Bajo esta premisa, se estudió el comportamiento frente a la corrosión en medios de exposición de $\mathrm{NaCl}$ (3\%) y $\mathrm{H}_{2} \mathrm{SO}_{4}(5 \%)$. El objetivo era presentar un ejemplo para la adecuada selección de materiales que presenten apropiado comportamiento cuando esté sometido a estos ambientes. 


\section{Materiales y métodos}

El proceso experimental llevado a cabo, se esquematiza en la Figura 1. Como paso inicial, se prepararon ocho (08) probetas de cada uno de los materiales con dimensiones de máximo $1.5 \mathrm{~cm}$ x $2.5 \mathrm{~cm}$ y entre $0.4 \mathrm{~cm}$ y $0.6 \mathrm{~cm}$ de espesor. Para el caso de los aceros inoxidables empleados, fue necesario realizar un pulido mecánico con lijas 80 y 120 para desbaste basto. Lijas 240,300 y 400 para desbaste medio y lija 600 para desbaste fino. Luego de lijadas, se llevaron a pulido espejo utilizando una pulidora de paños a la que previamente se le aplicó alúmina. El proceso para el caso del dióxido de zirconio no sinterizado, se redujo al corte del material, y su posterior alisamiento superficial mediante el uso de una lija para desbaste fino.

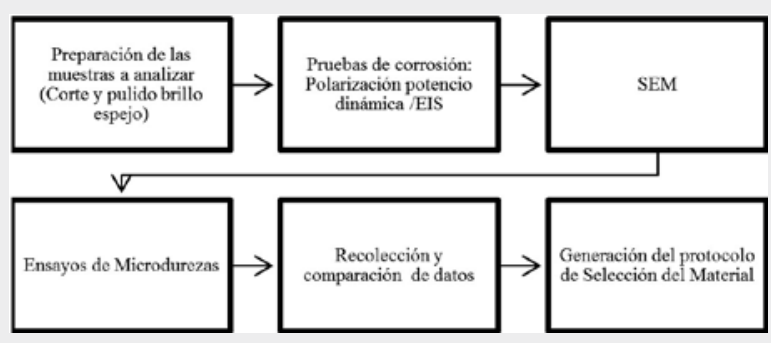

Figura 1. Esquema de actividades.

Fuente: Autores.

Una vez que las probetas fueron llevadas a acabado espejo, fueron pesadas y se les tomó lecturas de micro dureza. Para la medición de microdureza, se utilizó la escala Vickers, realizando cuatro (4) mediciones en grupo por cada probeta utilizando cargas de $500 \mathrm{~g}$ en el equipo. La dureza Vickers se obtuvo de la Ecuación 1:

$$
H_{v}=\frac{1.8544 F}{D^{2}}
$$

\section{Donde;}

Hv es la dureza expresada en Vickers, F es la carga aplicada sobre la muestra $D$ es la longitud de las diagonales del indentador.
Los ensayos de corrosión se realizaron mediante pruebas potenciodinámicas o extrapolación Tafel y espectroscopia de impedancia electroquímica EIS, usando un equipo GAMRY Reference 600 Potenciostat/ Galvanostat/ZRA. Para ello, se utilizó un electrodo de referencia de calomel saturado (SCE) y un contraelectrodo de grafito de alta pureza, ajustándose a las recomendaciones de la norma ASTM G5 [12]. Se llevaron a cabo dos sistemas de medio exposición, el primero fue una solución de $\mathrm{NaCl}$ al $3 \%$, con la que hizo contacto la muestra en un área de $0.59 \mathrm{~cm}^{2}$ y el segundo, fue una solución electrolítica de $5 \%$ H2SO4 [13], [14]. Los parámetros de barrido corresponden a un intervalo de $-0,3 \mathrm{~V}$ y $1 \mathrm{~V}$ con respecto al potencial de reposo, con una variación de $0,5 \mathrm{~m} \mathrm{~V} / \mathrm{s}$. Todas las muestras fueron sometidas a tiempo de reposo de una hora, previa realización del ensayo.

En este caso, se realizó un ajuste lineal de los datos de acuerdo con la aproximación de Tafel. Se construyó luego una tabla de corriente y potencial con el fin de representar $\log \mathrm{iT}=f(\eta)$ y obtener Icorr (corriente de corrosión) y por último, Ecorr(voltaje de corrosión).

En los ensayos electroquímicos se debe tener en cuenta que si la concentración de los reactivos y de los productos es uniforme en el electrolito, la ecuación de Butler-Volmer [13] toma la forma de la Ecuación 2:

$$
j=j_{0}\left[\exp \left(\frac{\eta_{a}}{\beta_{a}}\right)-\exp \left(\frac{\eta_{c}}{\beta_{c}}\right)\right]
$$

Dónde:

ISSN 0122-820X

PP: 112-124

$\beta_{a} \quad y \quad \beta_{c}$ son los coeficientes anódico $y$ catódico respectivamente y se denominan coeficientes Tafel.

$\eta_{a}$ y $\eta_{c}$ son los respectivos potenciales de activación del ánodo y el cátodo y $j_{0}$ es la corriente de activación. 
No. 1

Enero - Junio 2015 ISSN 0122-820X

PP: $112-124$

116
Para determinar experimentalmente los parámetros cinéticos $j_{0}, \beta_{a}$ y $\beta_{c}$ que son los coeficientes anódico y catódico respectivamente, se hace una representación gráfica en la que la densidad de corriente esté en escala logarítmica, poniendo en evidencia la relación lineal existente entre $\log$ I y el potencial de activación $\eta$, especialmente cuando su valor absoluto es grande. Luego de obtenida la curva de polarización experimental, la extrapolación de la recta en el dominio tafeliano al valor del potencial de equilibrio, se determina la densidad de corriente de intercambio. El inverso de la pendiente de las rectas, proporciona el valor de los coeficientes Tafel.

La técnica de espectroscopía de impedancia electroquímica (EIS) se basa en el uso de una señal de potencia aplicada sobre un electrodo, para medir la respuesta en corriente a diferentes frecuencias. Así el equipo electrónico empleado procesa las mediciones de potencial dando como resultado una serie de impedancias a cada frecuencia estudiada, conocido como espectro de impedancias. Estos espectros se analizan como un circuito eléctrico denominado "circuito equivalente".

Los diagramas de Bode se obtuvieron realizando barridos de frecuencia en el rango de $0.01 \mathrm{~Hz}$ hasta $100 \mathrm{kHz}$, empleando una amplitud de la señal sinusoidal de $10 \mathrm{mV}$, con un área de exposición de la muestra de $0.59 \mathrm{~cm}^{2}$. Un aspecto importante dentro de esta clase de ensayo de espectroscopia de impedancia electroquímica (EIS) es la obtenida dependiendo de los valores conseguidos en sus gráficas de impedancia y frecuencia como se muestra en la Figura 2. Los recubrimientos que tengan valores de impedancia, a una frecuencia de $0.1 \mathrm{~Hz}$, superiores a $108 \Omega . \mathrm{cm} 2$ se pueden clasificar de excelente protección, mientras que aquellos que tengan valores por debajo de $106 \Omega$.cm2 se clasifican como de pobre protección. Uno de los diagramas que nos permiten visualizar mejor este comportamiento es el diagrama de Bode [15], [16]. En los diagramas del módulo de impedancia versus frecuencia la presencia de una meseta horizontal se asocia a los elementos resistivos, ya que los valores de las resistencias no cambian con respecto a la frecuencia, mientras que las pendientes negativas se asocian a elementos capacitivos y las pendientes positivas están relacionadas a elementos inductivos. Los valores de la resistencia del electrolito y la transferencia de carga pueden visualizarse a altas y bajas frecuencias, respectivamente [17]. De igual manera, en el diagrama de fase se pueden establecer las frecuencias de formación de capacitores y los puntos de relajación, los cuales representan el momento en que se rompe la protección de la película pasiva debido a la probable penetración de electrolito y la consecuente disolución del recubrimiento. Un ángulo de $90^{\circ}$ representa un capacitor perfecto ya que este elemento eléctrico presenta un desfasamiento entre las señales de corriente y voltaje sinusoidales [18].

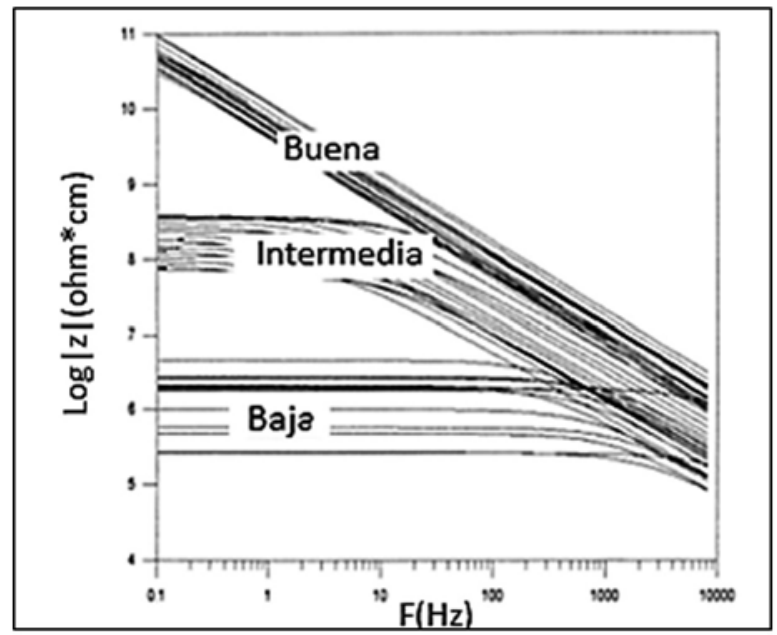

Figura 2. Grafica de clasificación de resistividad eléctrica mediante pruebas de impedancia electroquímica Fuente:[18]

Luego, se procedió a evaluar las características de la superficie de los depósitos de corrosión utilizando la técnica de microscopia electrónica de barrido (SEM), con el uso del equipo FEI QUANTA 200 en alto vacío y a un voltaje de $30 \mathrm{kV}$. Finalmente, se recolecto la información obtenida a fin de establecer el protocolo de selección del material idóneo para este ejemplo. 


\section{Resultados obtenidos}

\subsection{Ensayo de dureza}

Se realizaron 10 mediciones de indentaciones en distintas partes de las superficies de los materiales de estudio pre y post ensayo de corrosión a fin determinar la influencia del medio de exposición en esta propiedad; determinándose que no se presentaron cambios importantes en la dureza dado el tiempo de exposición de las muestras a los ambientes corrosivos (aproximadamente 1 hora). Ver Figura 3.

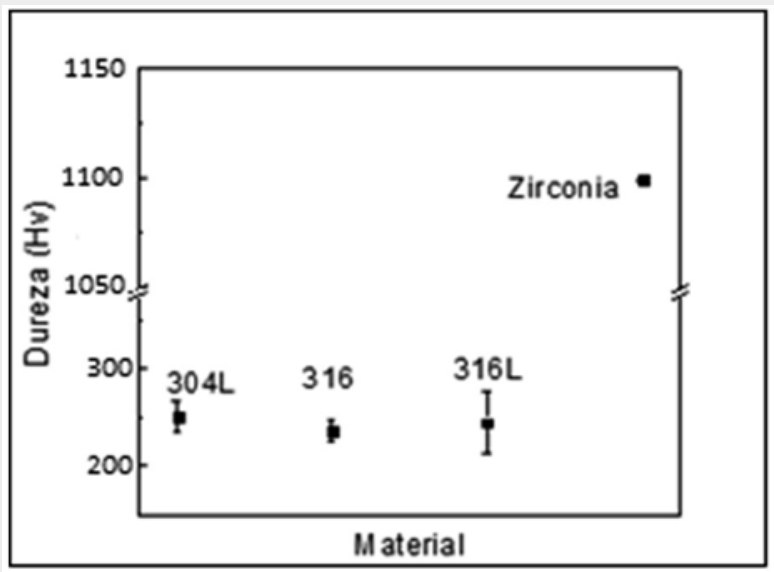

Figura 3. Microdurezas en los materiales de estudio.

Fuente: Autores

\subsection{Ensayos de corrosión}

Los ensayos de corrosión mediante espectrometría de impedancia electroquímica y polarización potenciodinámica se llevaron a cabo en dos soluciones: $\mathrm{NaCl}(3 \%)$ y $\mathrm{H}_{2} \mathrm{SO}_{4}$ $(5 \%)$ a temperatura ambiente.

En la Figura 4(A), se presentan los resultados del ensayo de extrapolación por Tafel para los materiales sometidos a la prueba con 5\% de ácido sulfúrico $\left(\mathrm{H}_{2} \mathrm{SO}_{4}\right)$. En este caso, el acero AISI 316 presenta menor cantidad de corriente de corrosión y una pasivación a mayor potencial, por lo tanto, es el material que ofrece mayor resistencia a la corrosión en este medio. De esto se deduce que dado el alto potencial de pasivación, en caso de circular corriente esta es más pequeña que la presentada por otros materiales, caso contrario, sucede en el material AISI 304L, en donde la densidad de corriente es mayor. También se aprecia en la región anódica la presencia de disolución de la superficie del material, sin embargo en el acero AISI 304 se observa la región de pasivación a altos valores de voltaje.

En la Figura 4(B) se presentan los resultados de la corriente de corrosión y el potencial de corrosión de los materiales analizados en medio electrolítico de $3 \% \mathrm{NaCl}$, se observa que los valores de potencial de corrosión más positivos se presentan en los aceros inoxidables, además se evidencia mejor comportamiento, es decir una menor velocidad de corrosión en los aceros de la serie 316 , debido posiblemente a que la película formada por la pasivación de la superficie tiene mejores propiedades electroquímicas. Mientras que en el caso del material cerámico es posible que la porosidad $y$ defectos generados del proceso de síntesis aumenten la velocidad de corrosión. A fin de corroborar los resultados descritos cualitativamente para las curvas de polarización potenciodinámica, se muestran los datos determinados por el programa GAMRY Reference 600 para la densidad de corriente de corrosión (Icorr), La velocidad de corriente de corrosión (Vcor) y los coeficientes Tafel. El error se calcula evaluando el Icor y Vcor para tres (03) puntos distintos.

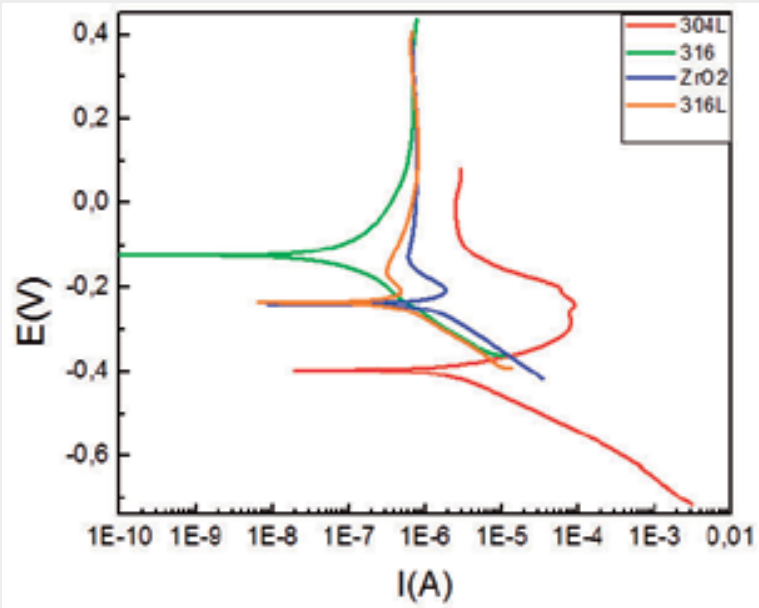

117

(A) 


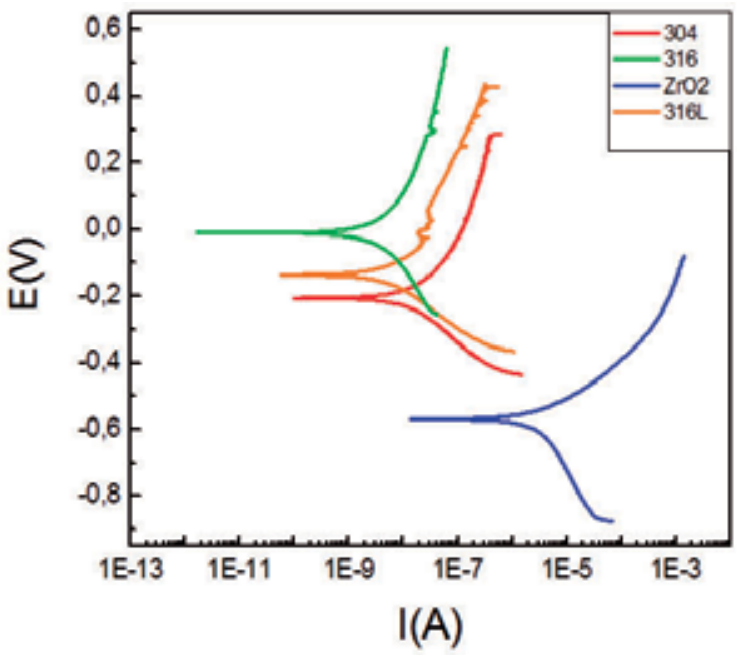

(B)

Figura 4. (A) Curvas de polarización potencio dinámicas materiales de estudio en $\mathrm{H}_{2} \mathrm{SO}_{4}$. (B) curvas de polarización potencio dinámicas de los materiales de estudio en $\mathrm{NaCl}$. E, representa el voltaje.

Fuente: autores

Tabla I. (A) Datos calculados con electrolito $\mathrm{NaCl},(B)$ Datos calculados con electrolito $\mathrm{H}_{2} \mathrm{SO}_{4}$

\begin{tabular}{|c|c|c|c|c|}
\hline NaCl & $\begin{array}{c}\text { Icorr } \\
(\mathbf{A}) \times \mathbf{x 1 0}^{-8}\end{array}$ & Ecorr $(\mathbf{m V}) \times-0^{-1}$ & BetaA & BetaC \\
\hline ZrO2 & $34 \pm(7)$ & -5.69 & $0.11 \pm(0.01)$ & $0.3 \pm(0.1)$ \\
\hline 304L & $2.07 \pm(0.05)$ & -2.05 & $\begin{array}{c}0.8236 \pm \\
(0.004)\end{array}$ & $0.201 \pm(0.01)$ \\
\hline 316 & $1.24 \pm(0.05)$ & -0.09 & $0.82 \pm(0.04)$ & $0.55 \pm(0.02)$ \\
\hline 316L & $0.9 \pm(0.4)$ & -1.39 & $0.3 \pm(0.2)$ & $0.15 \pm(0.03)$ \\
\hline
\end{tabular}

(A)

\begin{tabular}{|c|c|c|c|c|}
\hline $\mathbf{H}_{2} \mathbf{S O}_{4}$ & $\begin{array}{c}\text { Icorr } \\
(\mathbf{A}) \mathbf{x} \mathbf{0}^{\text {s }}\end{array}$ & $\begin{array}{c}\text { Ecorr } \\
(\mathbf{m V}) \mathbf{x}-0^{-1}\end{array}$ & BetaA & BetaC \\
\hline ZrO2 & $3 \pm(1)$ & -1.63 & $0.6 \pm(0.2)$ & $0.4 \pm(0.1)$ \\
\hline 304L & $21 \pm(1)$ & -3.96 & $\begin{array}{c}0.045 \pm \\
(0.03)\end{array}$ & $\begin{array}{c}0.088 \pm \\
(0.001)\end{array}$ \\
\hline 316 & $1.2 \pm(0.2)$ & -1.25 & $0.23 \pm$ & $0.14 \pm$ \\
\hline 316L & $5.9 \pm(0.1)$ & -2.3 & $12 \pm(7)$ & $0.17 \pm(0.08)$ \\
\hline
\end{tabular}

(B)

Fuente: Autores

De acuerdo con los resultados de la Tabla
I(A), los materiales más resistentes a la corrosión son los aceros 316 y 316L. En este caso estudio se consideran los dos, porque el margen de error de los aceros se superpone, haciendo que ambos materiales se encuentren en el mismo rango de corriente. El error en la medición en el caso del acero 316L se debe a las variaciones de corriente por posibles picaduras, tal como se evidencia en la Figura 2 , en el valor de voltaje cercano a cero y de corriente en el rango de $1 \times 10^{-8}$ y $1 \times 10^{-7}$.
Para el caso de la Tabla I (B), el material con menor valor de corriente y por tanto, mayor resistencia a la corrosión es el acero 316L. De este modo, tanto analítica como gráficamente y dentro del rango de error, el acero 316L es el material más adecuado para el caso de ambientes con cloro.

En las gráficas Figura 5(A-B) se muestran los diagramas de Bode en función del tiempo, indicando que un tiempo de relajación se encuentra en el rango de frecuencias $0.1-1 \mathrm{~Hz}$ para los materiales de estudio, en un medio $\mathrm{NaCl}$. El gráfico de impedancia $(Z)$ muestra un aumento con el tiempo, lo cual indica que la resistencia a la polarización $(\mathrm{Rp})$ aumenta debido a la generación de capas protectoras y/o disminución de la conducción de iones en la zona atacada por el electrolito. Los resultados muestran que a bajas frecuencias el valor de impedancia se incrementa con el paso del tiempo lo cual se repite para los cuatro (4) materiales, destacando que los aceros de la serie 316 presentan altos valores de impedancia sin un cambio significativo en la frecuencia, además se determina que el material cerámico necesita de un mayor intervalo de tiempo para estabilizarse y así generar mayores valores de resistencia a la polarización. El alto valor de impedancia del material cerámico en $0.01 \mathrm{~Hz}$ puede estar asociado a los mayores valores de resistencia del electrolito.

El valor del ángulo de fase se incrementa en función del tiempo del ensayo, lo que muestra que durante el tiempo se generaron capas protectoras en las zonas afectadas por el medio de exposición o se taponaron poros con productos de corrosión; además se observa que para cada uno de los materiales analizados en la región media del diagrama existe un señal que puede estar asociada a dos tiempos de relación típicas de superficies porosas con altos valores del ángulo de fase, a partir de lo cual se puede decir que la superficie de 
los materiales actúa como un buen capacitor que almacena carga, es decir, retarda la transferencia de carga del electrolito hacia la superficie del material; generando una capa protectora (Ver Figura 5 B).

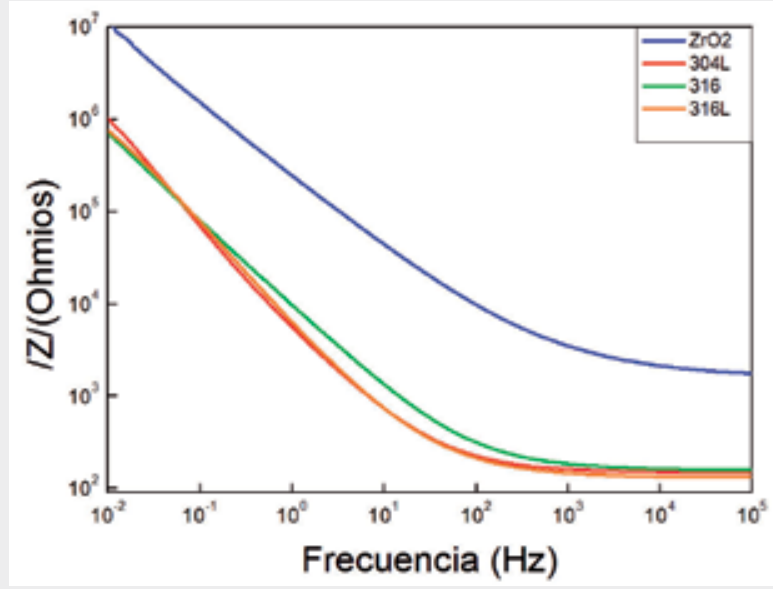

(A)

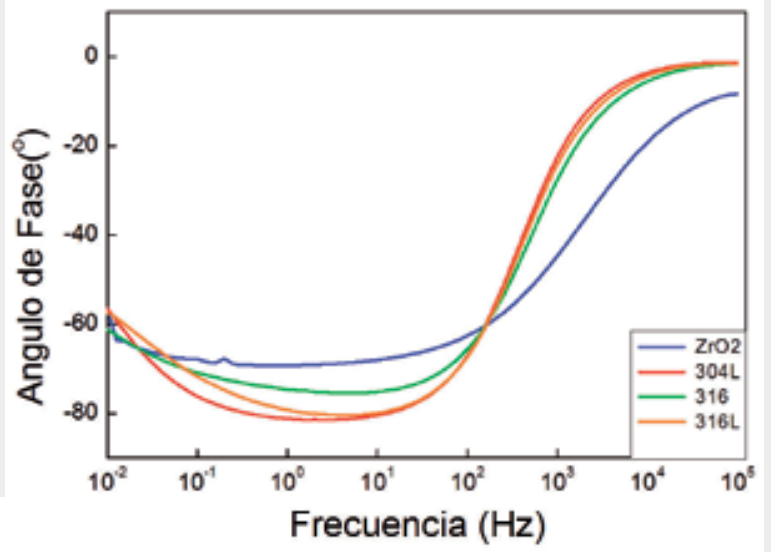

(B)

Figura 5. Gráficas de bode para los materiales estudiados con medio de exposición $\mathrm{NaCl}$ (A) Impedancia vs Frecuencia. (B) Ángulo de fase vs Frecuencia.

Fuente: Autores

Con respecto a los datos obtenidos en el medio $\mathrm{H}_{2} \mathrm{SO}_{4}$ de la Figura 6(A), el grafico de Bode muestra un aumento con la disminución de la frecuencia para los materiales ferrosos, mientras que para el material cerámico se obtiene un comportamiento parabólico con valores de impedancia pequeños a lo largo del eje de las frecuencias, esto indica que el material no generó interfaces efectivas de protección.

En el diagrama del ángulo de fase, Figura 6
(B), se muestran dos constantes de tiempo parcialmente definidas para el material cerámico a un bajo valor del ángulo de fase, lo que indica que se están generando nuevos elementos de transferencia de carga, responsables de permitir que el electrolito alcance la superficie del sustrato, mientras que para los materiales ferrosos al igual que en el caso de medio $\mathrm{NaCl}$, los tiempos de relajación se presentan con un alto valor del ángulo de fase, que puede ser explicado por las buenas propiedades electroquímicas de la película pasiva que se ha formado [19] .

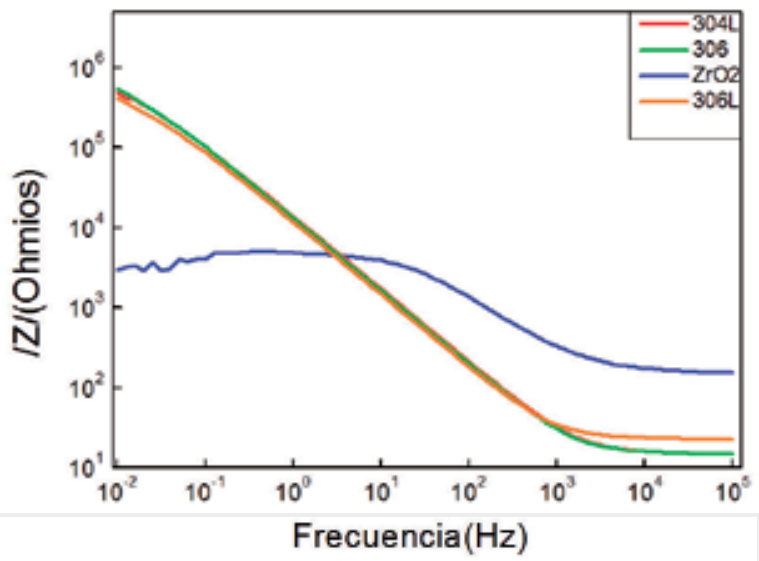

(A)

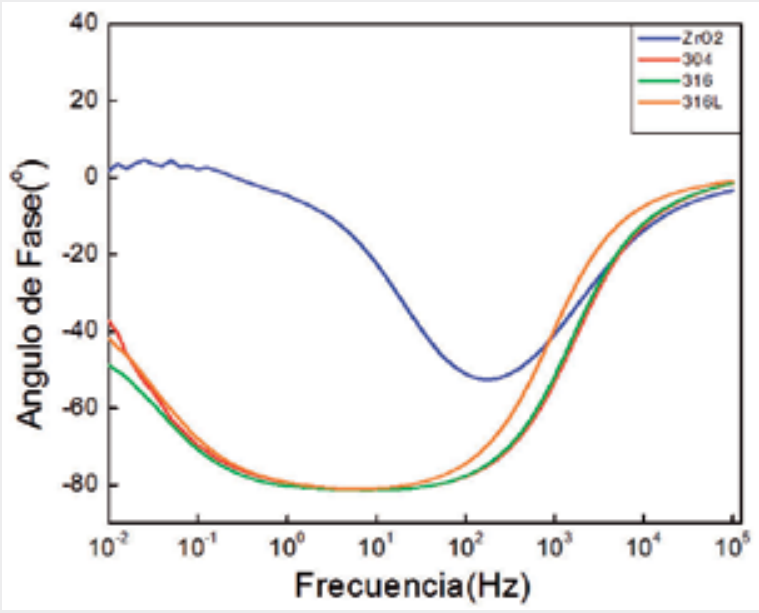

(B)

\footnotetext{
Figura 6. Gráficas de bode para los materiales estudiados con medio de exposición $\mathrm{H}_{2} \mathrm{SO}_{4}(\mathrm{~A})$ Impedancia vs Frecuencia. (B) Ángulo de Fase vs Frecuencia.

Fuente: Autores
}

En las Figura7(A-B-C) se observan la morfología de la superficie de los materiales de estudio después de algunos ensayos corrosión, 
Aplicación de una metodología mixta para la selección de materiales resistentes a la corrosión en medios ácidos y salinos

como lo son la generación de defectos o daños en la muestra del acero AISI 316 y 316L, así como la generación de productos de corrosión apreciables en el acero AISI 314L sometido en un electrolito $\mathrm{H}_{2} \mathrm{SO}_{4}$. En general, estos productos de corrosión se producen por la difusión de electrolito corrosivo a través de los defectos de la capa protectora del acero inoxidable hasta la superficie metálica.

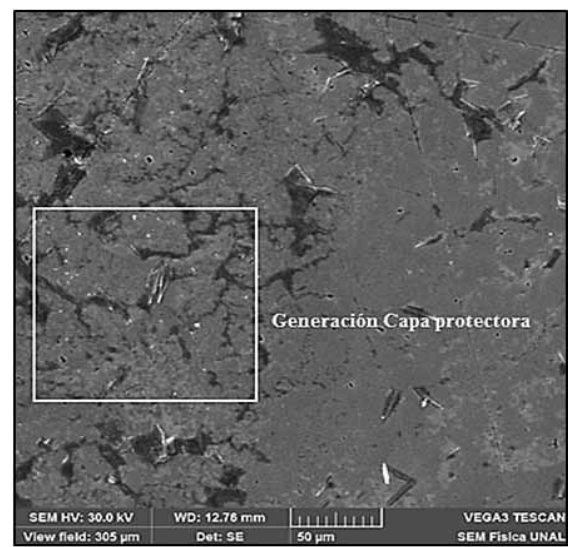

(A)

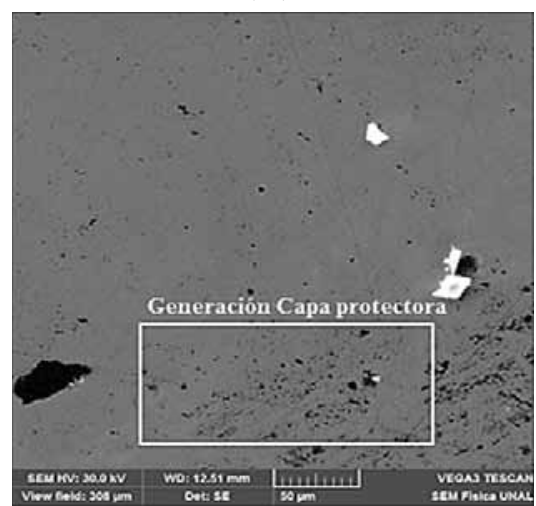

(B)

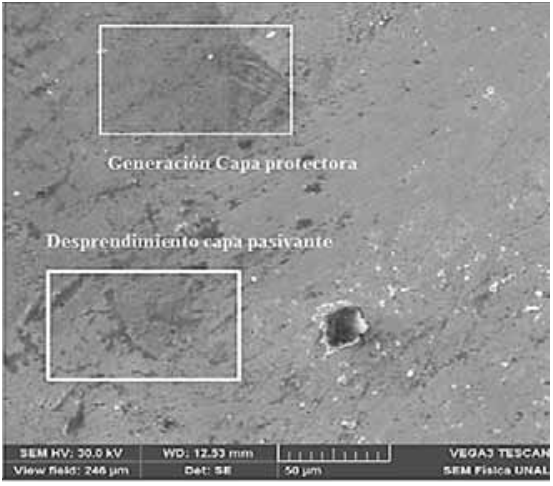

(C)

Figura 7. Micrografías SEM materiales expuestos a electrolito $\mathrm{H}_{2} \mathrm{SO}_{4}$ (A) Acero AISI 316. Evidencia de formación de capa protectora. (B) Acero AISI 316L.Evidencia de capa protectora. (C) Acero AISI 304L.Falta de adherencia de la capa protectora.

\subsection{Método de Selección}

Se aplica metodología de Ashby [10], [11], en donde se usa un índice de desempeño que generalmente hace parte de una función objetivo, la cual maximiza o minimiza un atributo del material. Siguiendo cada uno de los pasos propuestos en la misma (Ver Tabla II):

Materiales con buenas propiedades mecánicas y químicas (modulo elástico, resistencia a la corrosión) elevadas durezas, resistencia a la corrosión en medios marinos y ácidos y, bajo costo.

Tabla II. Restricciones de diseño

\begin{tabular}{|c|l|}
\hline \multicolumn{2}{|c|}{ REQUISITOS } \\
\hline Función & Material con buenas propiedades anticorrosivas \\
\hline \multirow{2}{*}{ Restricciones } & No fallar en medios corrosivos \\
\cline { 2 - 2 } & Bajo costo, fácil conformado. \\
\hline Objetivo & $\begin{array}{l}\text { Maximizar propiedades mecánicas y resistencia a la } \\
\text { corrosión, minimizar costos }\end{array}$ \\
\hline Variables Libres & Escogencia del material \\
\hline
\end{tabular}

Fuente: Autores

Los índices de desempeño vienen dados por las relaciones entre propiedades mecánicas de micro dureza y módulo elástico, además de la resistencia a la corrosión y la restricción de bajo costo. Inicialmente se tiene que la dureza se define como la relación existente entre la fuerza ejercida por el indentador dividida por el valor de la diagonal de la huella al cuadrado, ver Ecuación (3).

$$
H=\frac{1.854 * F}{A^{2}}
$$

Dónde:

\section{F: Carga Aplicada}

A: longitud de la diagonal del cuadrado de la impresión

Esta, Ecuación (4) se relaciona con el valor, así que se tiene [11]:

$$
H \approx 3 \sigma_{y}
$$

Donde; 


$$
\sigma_{y}: \text { Esfuerzo de fluencia }
$$

Donde;

Por lo que se tiene que el primer índice viene dado en función de la Ecuación 5:

$$
\left.\mathbf{M}_{1}=\sigma_{y} \quad 5\right)
$$

El material debe ser duro pero capaz de deformarse dentro de cierto intervalo de esfuerzos antes de fallar. La dureza se relaciona con el módulo elástico mediante Ecuación 6 [20], en donde se consideran de forma explícita tanto la influencia del ángulo entre el indentador y el material, como el efecto de la elasticidad mediante el módulo de Young, E.

$$
H / \sigma=1.44+0.264 \operatorname{In}(E / \varepsilon)
$$

Donde la dureza, $H$, es directamente proporcional a dicha tensión representativa, $\sigma$ que puede ser evaluada directamente en la curva de tracción a un valor de deformación. Por lo tanto la Ecuación 7:

$$
\mathbf{M}_{2}=(7)
$$

Al minimizar costos se tiene la Ecuación 8:

$$
\mathbf{M}_{3}=(8)
$$

Cm: Costo $(\$ / K g)$

Los valores obtenidos, y los materiales de estudio se clasifican en un orden del más alto al más bajo en relación a las propiedades objeto de estudio.

Tabla III. Valores índices materiales

\begin{tabular}{|c|c|c|c|}
\hline MATERIAL & $\mathbf{M}_{\mathbf{1}}(\mathrm{MPa})$ & $\mathbf{M 2}(\mathrm{GPa})$ & OBSERVACIONES \\
\hline ACEROS DE BAJO CARBONO & $70-150$ & $8-12$ & Bajo costo \\
\hline ACEROS INOXIDABLES 316 & $210-230$ & $14-24,2$ & $\begin{array}{c}\text { Aplicaciones } \\
\text { a altas } \\
\text { temperaturas, } \\
\text { buen candidato. }\end{array}$ \\
\hline ACEROS INOXIDABLES 304L INOXIDABLES 316L & $200-215$ & $14-25$ & $\begin{array}{c}\text { Aplicaciones } \\
\text { biomédicas, buen } \\
\text { candidato }\end{array}$ \\
\hline CARBURO DE TUGSTENO & 550 & $17-24$ & $\begin{array}{c}\text { Alta dureza, alto } \\
\text { precio }\end{array}$ \\
\hline ZIRCONIA NO SINTERIZADA & 138 & 11 & $\begin{array}{c}\text { Difícil obtención, } \\
\text { tiene que estar } \\
\text { estabilizada }\end{array}$ \\
\hline ALEACIONES TITANIO & $400-600$ & $25-50$ & $\begin{array}{c}\text { Alto precio, } \\
\text { excelentes } \\
\text { propiedades } \\
\text { mecánicas }\end{array}$ \\
\hline
\end{tabular}

Fuente: Autores
No. 1

Enero - Junio 2015

ISSN 0122-820X

PP: $112-124$

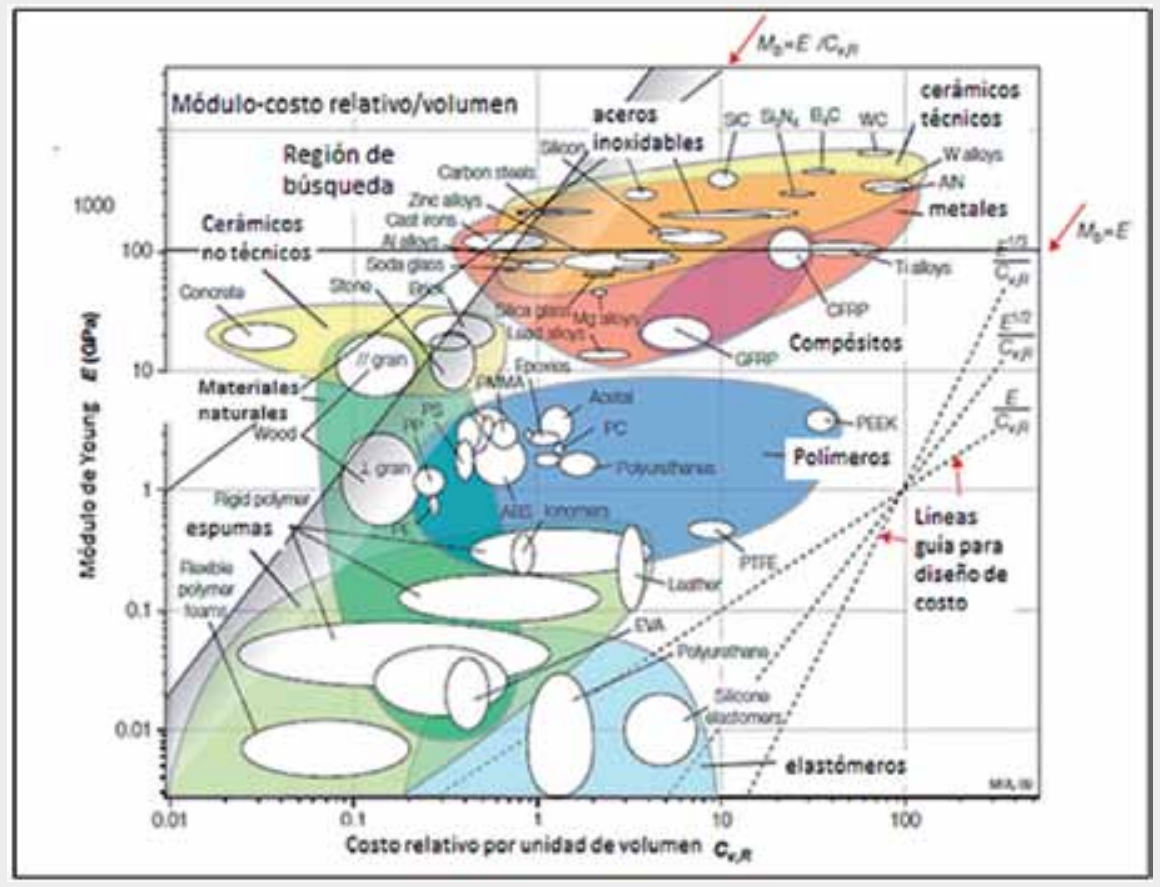

Figura 8. Materiales con bajo costo y elevadas propiedades mecánicas Fuente: [11] (adaptado) 
No. 1

Enero - Junio 2015 ISSN 0122-820X

PP: $112-124$

122
Al observar la Figura 8 y los valores de los parámetros obtenidos se denota que los mejores materiales que cumplen con las restricciones dadas son los que se ubican sobre una recta que va desde 1 hasta $10(\$ /$ $\mathrm{Kg}$ ) en el eje $\mathrm{x}$ con módulos elásticos entre un rango de 100 y $500 \mathrm{GPa}$ del gráfico de Ashby (costo/modulo elástico). En la Tabla III se muestra los valores de los índices a maximizar de posibles candidatos, en donde se puede observar que la mejor opción se basa en los materiales con valores de módulos elásticos cercanos a los $200 \mathrm{GPa}$, valores de límites elásticos cercanos a los $20 \mathrm{GPa}$.

Utilizado la metodología mixta: Ashby versus resultados experimentales y con ayuda de bases de datos, se logró determinar los valores promedios de la resistividad de algunos materiales como se muestra en la Tabla IV [21], [22], a partir de estos valores, y con los resultados obtenidos mediante el método gráfico, se determina el mejor material para este tipo de aplicaciones es el acero AISI 316; el cual debido a su contenido de carbono, presenta un mayor límite elástico, dureza y menor resistencia a la tracción [23].

\begin{tabular}{|l|c|c|}
\hline \multicolumn{2}{|c|}{ Tabla IV. Resistividad de algunos materiales } \\
\hline Material & $\begin{array}{c}\text { Resistividad } \\
(* \mathrm{~cm})\end{array}$ & Observaciones \\
\hline $\begin{array}{l}\text { Aceros de bajo } \\
\text { carbono }\end{array}$ & $15-20$ & $\begin{array}{c}\text { Buenos candidatos por su alta tasa } \\
\text { de pasivasión }\end{array}$ \\
\hline Aceros AISI 316 & $70-78$ & Buen candidato \\
\hline Aceros AISI 316L & $70-107$ & Buen candidato aunque costoso \\
\hline Aceros AISI 304L & 72 & Buen candidato también costoso \\
\hline Cobre & $1.7-2.4$ & Alta corriente de corrosión \\
\hline Zirconia & 44 & Poca resistencia a la fractura \\
\hline $\begin{array}{l}\text { Aleaciones } \\
\text { Titanio }\end{array}$ & $100-170$ & Pueden no ser biocompatibles \\
\hline Cauchos & $10^{13}-10^{16}$ & Baja dureza y poca resistencia a alta \\
temperatura
\end{tabular}

Fuente: [21]

\section{Conclusiones}

La selección correcta de un material depende de una gran cantidad de factores, pudiéndose llegar a una aproximación muy genérica, si no se cuenta con los índices adecuados. Ahora bien, comparando los valores obtenidos mediante esta metodología de selección de materiales y los obtenidos mediante experimentación se comprueba que la familia de materiales estudiados puede ser aplicada en ambientes corrosivos.

Dentro del grupo de materiales presentados en este artículo, las aleaciones metálicas AISI 316 presentaron un buen comportamiento en los dos medios de estudio $\mathrm{NaCl} / \mathrm{H}_{2} \mathrm{SO}_{4}$; además al consultar la bibliografía se obtiene que este material posee mejores propiedades mecánicas comparadas con los otros materiales del estudio, lo cual lo convierte en una buena opción para las aplicaciones severas de corrosión.

Si bien la Zirconia se adecuada a las restricciones dadas, es un cerámico que como material en bulto no permite generar elementos funcionales compuesto en su totalidad, por lo cual generalmente, debe estar sinterizado para disminuir defectos como poros para mejorar su resistencia a la corrosión.

\section{Referencias}

[1] H. F. Gomez de Leon, L. D. Alcaraz. Manual Básico de corrosión para ingenieros. Universidad de Muercia. 2004.

[2] J. V.Osuna, L.A. Gonzalez, M.C. Beltran, B. Valdez, "Estrategia administrativa para la auto sustentabilidad de centros de investigación en instituciones de educación superior", Administración Contemporánea, vol. 6, no. 21, pp. 7694, mayo de 2014.

[3] Universia Colombia. Más de 26 mil millones de pesos pierde la industria colombiana debido a la corrosión de materiales. Agosto 2013. [Online]. Disponible en: http:// noticias.universia.net.co/actualidad/ 
noticia/2013/08/29/1045848/mas-26mil-millones pesos-pierde-industriacolombiana-debido-corrosionmateriales.html.

[4] Euro Inox. Decapado y Pasivado del acero inoxidable. Serie Materiales y sus Aplicaciones, Volumen 4. 2004. [Online]. Disponible en: http://www. euro-inox.org/pdf/map/Passivating Pickling_SP.pdf.

[5] E. P. DeGarmo, J. T. Black, R. A. Kosher. Materiales y Procesos de Fabricación. Volumen 1. Barcelona: Editorial Reverté S.A, 2002, p. 208.

[6] P.Corrado, G. Saverio, K. Tomaž. "Chapter 11-Alumina- and Zirconiabased Ceramics for Load-bearing Applications", en Advanced Ceramics in Dentistry, Elsevier 2014, pp. 220230 .

[7] Y. Zhou. Welding in energy-related projects. Pergamon Press Cánada Ltda, 1984, pp. 209-211.

[8] F. C. Nascimento, C. E. Foerster, S. Rutz Da Silva, C. M. Lepienski, C. J. De Mesquita Siqueira, C. Alvez, "A Comparative study of mechanical and tribological properties of AISI-304 and AISI-316 submitted to glow discharge nitriding", Material Research, vol. 12, no. 2, pp. 173-180, 2009.

[9] R. L. Plaut, C. Herrera, D. M. Escriba, P. Rangel Rios, A. F. Padilha, "A short review on wrought austenitic stainless steels at high temperatures: processing, microstructure, properties and performance", Material Research, vol. 10, no. 4, pp. 453-460, 2007.

[10] M. F. Ashby. Materials Selection in Mechanical Design. Third Edition. Elsevier. 2005.
[11] M. F. Ashby, K. Johnson. Material and Desing: The art and science of material selection in product. Third Edition. Elsevier. 2014.

[12] ASTM G5 - 94. Standard Reference Test Method for Making Potentiostatic and Potentiodynamic Anodic Polarization Measurements. 2004.

[13] Y. L. Chipateuica. Resistencia a la corrosión de multicpasnanometricas de $\mathrm{CrNx} / \mathrm{Cr}$ depositadas con magnetrón desbalanceado. Tesis maestría. Universidad Nacional de Colombia. 2010.

[14] J. J. Olaya, U. Piratoba, S. E. Rodhil, "Resistencia a la corrosión de recubrimientos de $\mathrm{CrN}$ depositados por PVD con UBM: Tecnología eficiente y ambientalmente limpia", Revista Latinoamericana de Metalurgia $y$ Materiales, vol. 31, no. 1, pp. 44-51, 2011.

[15] X. Liu, J. Xiong, LV. Yongwu, Y. Zuo, "Study on corrosion electrochemical behavior of several different coating systems by EIS", Progress in Organic Coatings, vol. 64, no. 4, pp. 497-503, 2009.

[16] A. A. Guzmán, "Evaluación de la resistencia a la corrosión de sistemas de recubrimientos con altos sólidos por medio de espectroscopía de impedancia electroquímica", Tesis de Maestría, Universidad Nacional de Colombia, 2011. ISSN 0122-820X PP: $112-124$

17] K. S. Harscha. Principles of vapor deposition of thin films. Elsevier Ltd. 2006.

[18] C. C. Lee, F. Mansfeld, "Automatic classification of polymer coating quality using artificial neural networks", Corrosion Science, vol. 41, no. 3, pp. 439-461, 1998. 
[19] D. Klotz, "Characterization and Modeling of Electrochemical Energy Conversion Systems", Tesis Doctoral, Karlsruhe Institute of Technology (KIT), 2012.

[20] A. Mata, M. Anglada, J. Alcalá. "Ecuaciones de dureza para la caracterización de metales mediante identanción puntiaguda". En VIII Congreso Nacional de Propiedades Mecánicas de Sólidos, Valencia, España, pp. 513-522, Junio 2002.

[21] P. A. Tipler, G. Mosca. Física para la ciencia y la tecnología. Volumen 2. Editorial Revérte. 2007.

[22] A. A. Aymen, M. B. Mansour, W. Manfred, W. Lothar, "Effect of surface and bulk plastic deformations on the corrosion resistance and corrosion fatigue performance of AISI 316L", Surface and Coatings Technology. Vol. 259, Part C, pp. 448-455, 2014.

[23] North American Stainless. [Online]. Disponible en: http://www. northamericanstainless.com/wpcontent/uploads/2010/10/Grade-316316L1.pdf 